\title{
From euclidean field theory to quantum field theory
}

\author{
Dirk Schlingemann \\ II. Institut für Theoretische Physik \\ Universität Hamburg \\ and \\ The Erwin Schrödinger International Institute \\ for Mathematical Physics (ESI) \\ Vienna
}

\begin{abstract}
In order to construct examples for interacting quantum field theory models, the methods of euclidean field theory turned out to be powerful tools since they make use of the techniques of classical statistical mechanics.

Starting from an appropriate set of euclidean $n$-point functions (Schwinger distributions), a Wightman theory can be reconstructed by an application of the famous Osterwalder-Schrader reconstruction theorem. This procedure (Wick rotation), which relates classical statistical mechanics and quantum field theory, is, however, somewhat subtle. It relies on the analytic properties of the euclidean $n$-point functions.

We shall present here a $\mathrm{C}^{*}$-algebraic version of the OsterwalderScharader reconstruction theorem. We shall see that, via our reconstruction scheme, a Haag-Kastler net of bounded operators can directly be reconstructed.

Our considerations also include objects, like Wilson loop variables, which are not point-like localized objects like distributions. This point of view may also be helpful for constructing gauge theories.
\end{abstract}




\section{Introduction}

Why euclidean field theory? During the last two decades it turned out that the techniques of euclidean field theory are powerful tools in order to construct quantum field theory models. Compared to the method of canonical quantization in Minkowski space, which, for example, has been used for the construction of $P(\phi)_{2}$ and Yukawa 2 models [10, 11, 12, 14, 21, 22], the functional integral methods of euclidean field theory simplify the construction of interactive quantum field theory models.

In particular, the existence of the $\phi_{3}^{4}$ model as a Wightman theory has been established by using euclidean methods [5, 24, 18] combined with the famous Osterwalder-Schrader reconstruction theorem [19]. For this model the methods of canonical quantization are much more difficult to handle and lead by no means so far as euclidean techniques do. Only the proof of the positivity of the energy has been carried out within the hamiltonian framework [10, 13].

One reason why the functional integral point of view simplifies a lot is that the theory of classical statistical mechanics can be used. For example, renormalization group analysis [9] and cluster expansions [2] can be applied in order to perform the continuum and the infinite volume limit of a lattice regularized model. Instead of working with non-commutative objects, one considers the moments

$$
\mathfrak{S}_{n}\left(x_{1}, \cdots, x_{n}\right)=\int \mathrm{d} \mu(\phi) \phi\left(x_{1}\right) \cdots \phi\left(x_{n}\right)
$$

of reflexion positive measures $\mu$, usually called Schwinger distributions or euclidean correlation functions, on the space of tempered distributions.

Heuristically, the functional integral point of view leads to conceptionally simple construction scheme for a quantum field theory. Starting from a given lagrangian density $L$, the measure $\mu$ under consideration is simply given by

$$
\mathrm{d} \mu(\phi)=Z^{-1} \bigotimes_{x \in \mathbb{R}^{d}} \mathrm{~d} \phi(x) \exp \left(-\int \mathrm{d} x L(\phi(x), \mathrm{d} \phi(x))\right)
$$

where the factor $Z^{-1}$ is for normalization. Therefore, the lagrangian $L$ can be interpreted as a germ of a quantum field theory. Moreover, this also leads to a nice explanation of the minimal action principle. However, to give the expression above a rigorous mathematical meaning is always accompanied with serious technical difficulties. 


\section{Some comments on the Osterwalder-Schrader reconstruc-} tion theorem. In order to motivate the main purpose of our paper, we shall make some brief remarks on the Osterwalder-Schrader reconstruction theorem [19] which relates Schwinger and Wightman distributions. Let $T(S)$ be the tensor algebra over the space of test functions $S$ (in $\mathbb{R}^{d}$ ) and let us denote by $J_{E}(E$ stands for euclidean) the twosided ideal in $T(S)$, which is generated by elements $f_{1} \otimes f_{2}-f_{2} \otimes f_{1} \in$ $T(S)$ where $f_{1}$ and $f_{2}$ have disjoint supports. We build the algebra $T_{E}(S):=T(S) / J_{E}$ and take the closure $T_{E}^{\mathcal{T}}(S)$ of it in an appropriate locally convex topology. We claim that the euclidean group $\mathrm{E}(d)$ acts naturally by automorphisms $\left(\alpha_{g}, g \in \mathrm{E}(d)\right)$ on $T_{E}^{\mathcal{T}}(S)$.

A linear functional $\eta \in T_{E}^{\mathcal{T}}(S)^{*}$ fulfills the Osterwalder-Schrader axioms if the following conditions hold:

(EO) $\eta$ is continuous and unit preserving: $\langle\eta, \mathbf{1}\rangle=1$.

(E1) $\eta$ is invariant under euclidean transformations: $\omega \circ \alpha_{g}=\omega$.

(E2) $\eta$ is reflexion positive: The sesqui-linear form $a \otimes b \mapsto<\eta, \iota_{e}\left(a^{*}\right) b>$ is a positive semi-definite on those elements which are localized at positive times with respect to the direction $e \in S^{d-1}$ where $\iota_{e}$ is the automorphism which corresponds to the reflexion $e \mapsto-e$.

Given a linear functional $\eta$ which satisfies the conditions (EO) to (E2), the analytic properties of the distributions

$$
\mathfrak{S}_{n}\left(f_{1}, \cdots, f_{n}\right):=<\eta, f_{1} \otimes \cdots \otimes f_{n}>
$$

and $S_{n}\left(\xi_{1}, \cdots, \xi_{n}\right)=\mathfrak{S}_{n+1}\left(x_{0}, \cdots, x_{n}\right) ; \xi_{j}=x_{j+1}-x_{j}$

lead to the result:

Theorem 1.1 : There exists a distribution $\tilde{W}_{n} \in S^{\prime}\left(\mathbb{R}^{n d}\right)$ supported in the $n$-fold closed forward light cone $\left(\bar{V}_{+}\right)^{n}$ which is related to $S_{n}$ by the Fourier-Laplace transform:

$$
S_{n}(\xi)=\int \mathrm{d}^{n d} q \exp \left(-\xi^{0} q^{0}-\mathrm{i} \vec{\xi} \vec{q}\right) \tilde{W}_{n}(q)
$$

The proof of this Theorem 19] relies essentially on the choice of the topology $\mathcal{T}$. It does not apply for the ordinary $S$-topology, i.e. it is not enough to require that the $\mathfrak{S}_{n}$ 's are tempered distributions. This was stated wrongly in the first paper of [19] and was later corrected in the second one. We claim that, nevertheless, the Theorem might 
be true for the ordinary $S$-topology, but, at the moment, there is no correct proof for it. These problems show that the relation between euclidean field theory and quantum field theory is indeed subtle.

In order to formulate the famous Osterwalder-Schrader reconstruction theorem from a more algebraic point of view, we shall briefly introduce the notion of a local net and a vacuum state.

$\mathrm{P}_{+}^{\uparrow}$-covariant local nets: $\quad \mathrm{A} \mathrm{P}_{+}^{\uparrow}$-covariant local net of *-algebras is an isotonous 1 prescription $\underline{A}: \mathcal{O} \mapsto A(\mathcal{O})$, which assigns to each double cone $\mathcal{O}=V_{+}+x \cap V_{-}+y$ a unital *-algebra $A(\mathcal{O})$, on which the the Poincaré group $\mathrm{P}_{+}^{\uparrow}$ acts covariantly on $\underline{A}$, i.e. there is a group homomorphism $\alpha \in \operatorname{Hom}\left(\mathrm{P}_{+}^{\uparrow}, \operatorname{Aut} A\right)$, such that $\alpha_{g} A(\mathcal{O})=A(g \mathcal{O})$. Here $A$ denotes the ${ }^{*}$-inductive limit of the net $\underline{A}$. Furthermore, the net fulfills locality, i.e. if $\mathcal{O}, \mathcal{O}_{1}$ are two space-like separated regions $\mathcal{O} \subset \mathcal{O}_{1}^{\prime}$ then $\left[A(\mathcal{O}), A\left(\mathcal{O}_{1}\right)\right]=\{0\} . \quad \mathrm{A} \mathrm{P}_{+}^{\uparrow}$-covariant local net of $\mathrm{C}^{*-}$ algebras is called a Haag-Kastler net.

Vacuum states: A state $\omega$ on $A$ is called a vacuum state iff $\omega$ is $\mathrm{P}_{+}^{\uparrow}$-invariant (or translationally invariant), i.e. $\omega \circ \alpha_{g}=\omega$ for each $g \in \mathrm{P}_{+}^{\uparrow}$, and for each $a, b \in A$

$$
\int \mathrm{d} x<\omega, a \alpha_{(1, x)}(b)>f(x)=0
$$

for each test function $f \in S$ with $\operatorname{supp}(\tilde{f}) \cap \bar{V}_{+}=\emptyset$. This implies that there exists a strongly continuous representation $U$ of $\mathrm{P}_{+}^{\uparrow}$ on the GNS Hilbert space of $\omega$ such that

$$
U(g) \pi(a) U(g)^{*}=\pi\left(\alpha_{g} a\right)
$$

and the spectrum of $U(1, x)$ is contained in the closed forward light cone. Here $\pi$ is the GNS representation of $\omega$.

Usually it is required that a vacuum state $\omega$ is a pure state. This aspect is not so important for our purpose and we do not assume this here.

An example for a $\mathrm{P}_{+}^{\uparrow}$-covariant local net of *-algebras is given by the prescription

$$
\underline{T}_{M}(S): \mathcal{O} \longmapsto T_{M}(S(\mathcal{O}))
$$

\footnotetext{
${ }^{1}$ Isotony: $\mathcal{O}_{1} \subset \mathcal{O}_{2}$ implies $A\left(\mathcal{O}_{1}\right) \subset A\left(\mathcal{O}_{2}\right)$.
} 
where $T_{M}(S):=T(S) / J_{M}$ 巳 is the well known Borchers-Uhlmann algebra. We should mention here that now the test functions in $S$ are test functions in Minkowski space-time.

Let $\tau \in \operatorname{Hom}\left(\mathrm{P}_{+}^{\uparrow}, \operatorname{GL}(S)\right)$ be the action of the Poincaré group on the test functions which is given by $\tau_{g} f=f \circ g^{-1}$ then

$$
\alpha_{g}\left(f_{1} \otimes \cdots \otimes f_{n}\right):=\tau_{g} f_{1} \otimes \cdots \otimes \tau_{g} f_{n}
$$

defines a covariant action of $\mathrm{P}_{+}^{\uparrow}$ on $\underline{T}_{M}(S)$. Now, the theorem above leads to the famous Osterwalder-Schrader reconstruction theorem:

Theorem 1.2 : Given a linear functional $\eta$ which satisfies the conditions (E0) to (E2), then there exists a vacuum state $\omega_{\eta}$ on the Borchers algebra $T_{M}(S)$ such that

$$
<\omega_{\eta}, f_{1} \otimes \cdots \otimes f_{n}>=\mathfrak{W}_{n}\left(f_{1}, \cdots, f_{n}\right)
$$

where $\mathfrak{W}_{n}$ is defined by

$$
\mathfrak{W}_{n}(x)=\int \mathrm{d}^{n d} q \exp (-\mathrm{i} \xi q) \tilde{W}_{n}(q) \quad ; \quad \xi_{j}=x_{j+1}-x_{j}
$$

The fact that $\omega_{\eta}$ is a vacuum state on the Borchers algebra is completely equivalent to the statement that the distributions $\mathfrak{W}_{n}$ fulfill the Wightman axioms in its usual form (except the clustering)(see [25]).

\section{A heuristic proposal for the treatment of gauge theories.} As mentioned above, the main reason for using euclidean field theory is for constructing quantum field theory models with interaction. In four space time dimensions, the most promising candidates for interactive quantum field theory models are gauge theories. Scalar or multi-component scalar field theories of $P(\phi)_{4}$-type are less promising to describe interaction, since their construction either run into difficulties with renormalizability or, as conjectured for the $\phi_{4}^{4}$-model, they seem to be trivial [7].

The description of gauge theories within the Wightman framework leads to some conceptional problems. For example, in order to study

\footnotetext{
${ }^{2}$ The ideal $J_{M}$ ( $M$ stands for Minkowski) is the two-sided ideal in $T(S)$, which is generated by elements $f_{1} \otimes f_{2}-f_{2} \otimes f_{1} \in T(S)$ where $f_{1}$ and $f_{2}$ have space-like separated supports.
} 
gauge invariant objects in quantum electro dynamics one may think of vacuum expectation values of products of the field strength $F_{\mu \nu}$

$$
\mathfrak{W}_{\mu_{1} \nu_{1}, \cdots, \mu_{n} \nu_{n}}\left(x_{1}, \cdots, x_{n}\right)=\left\langle\Omega, F_{\mu_{1} \nu_{1}}\left(x_{1}\right) \cdots F_{\mu_{n} \nu_{n}}\left(x_{n}\right) \Omega\right\rangle
$$

which satisfy the Wightman axioms. Here, the problem arises when one wish to include fermions. For the minimal coupling one has to study correlation functions of the gauge field instead of those in the field strength. This leads to such well known problems as indefinite metric, solving constraints and so forth.

Moreover, there is another problem which we would like to mention here. Within the Wightman framework the quantized version of the gauge field $u_{\mu}$ is an operator valued distribution. On the other hand, the classical concept of a gauge field leads to the notion of a connection in a vector or principal bundle over some manifold which suggests to consider as gauge invariant objects Wilson loop variables

$$
w_{\gamma}[u]=\operatorname{tr}\left[\operatorname{Pexp}\left(\int_{\gamma} A\right)\right]
$$

and string-like objects

$$
s_{\gamma}[u, \psi]=\bar{\psi}(r(\gamma)) \operatorname{Pexp}\left(\int_{\gamma} u\right) \psi(s(\gamma))
$$

where $\psi$ is a smooth section in an appropriate vector bundle and $\gamma$ is an oriented path which starts at $s(\gamma)$ and ends at $r(\gamma)$.

Unfortunately, to express $w_{\gamma}(u)$ in terms of Wightman fields leads to difficulties. From a perturbation theoretical point of view one expects that the distribution $u$ is too singular in order to be restricted to a one-dimensional sub-manifold.

To motivate our considerations, we shall discuss here, heuristically, an alternative proposal which might be related to a quantized version of a gauge theory. It is concerned with the direct quantization of regularized Wilson loops

$$
w_{\gamma}(f)[u]=\int \mathrm{d} x \quad w_{\gamma+x}[u] f(x) .
$$

Here we allow $f \in E^{\prime}\left(\mathbb{R}^{d}\right)$ to be a distribution with compact support which has the form

$$
f(x)=f_{\Sigma}(x) \delta_{\Sigma}(x)
$$


where $\Sigma$ is a $d$-1-dimensional hyper-plane and $f_{\Sigma} \in C_{0}^{\infty}(\Sigma)$ and $\delta_{\Sigma}$ is the natural measure on $\Sigma$. We claim that such a type of regularization is necessary since in $d$-dimensional quantum field theories there are no bounded operators which are localized within $d$-2-dimensional hyperplanes [四].

Such a point of view has been discussed by J. Fröhlich [6], E. Seiler [23] or more recently by A. Ashtekar and J. Lewandowski [1].

In order to describe a quantum gauge theory in terms of regularized Wilson loop variables one wishes to construct a function $\gamma \mapsto \mathbf{w}_{\gamma}$ which assigns to each path $\gamma$ an operator valued distribution $\mathbf{w}_{\gamma}$ : $f \mapsto \mathbf{w}_{\gamma}(f)$, where the operators $\mathbf{w}_{\gamma}(f)$ are represented by operators on some Hilbert space $\mathcal{H}$. Heuristically, one expects that the operators $\mathbf{w}_{\gamma}(f)$ are unbounded [20].

(1) The operators $\mathbf{w}_{\gamma}(f)$ are self-adjoint for real-valued test functions with a joint core $\mathcal{D} \subset \mathcal{H}$

(2) w should transform covariantly under the action of the Poincaré group, i.e.

$$
\mathbf{w}_{g \gamma}\left(f \circ g^{-1}\right)=U(g) \mathbf{w}_{\gamma}(f) U(g)^{*} ; g \in \mathrm{P}_{+}^{\uparrow},
$$

where $U$ is a unitary strongly continuous representation of the Poincaré group on $\mathcal{H}$ and the spectrum of the translations is contained in the closed forward light cone $\bar{V}_{+}$.

(2) Moreover, the operators $\mathbf{w}_{\gamma}(f)$ should satisfy the locality requirement, i.e.

$$
\left[\mathbf{E}_{(\gamma, f)}\left(\Delta_{1}\right), \mathbf{E}_{\left(\gamma_{1}, f_{1}\right)}(\Delta)\right]=0
$$

if the (convex hulls) of the regions $\gamma+\operatorname{supp}(f)$ and $\gamma_{1}+\operatorname{supp}\left(f_{1}\right)$ are space-like separated. Here

$$
\mathbf{w}_{\gamma}(f)=\int \mathrm{d} \mathbf{E}_{(\gamma, f)}(\lambda) \lambda
$$

is the spectral resolution of $\mathbf{w}_{\gamma}(f)$

According to [6, 23], it has been suggested to reconstruct Wilson loop operators $\mathbf{w}_{\gamma}$ from euclidean correlation functions of loops

$$
\gamma_{1}, \cdots, \gamma_{n} \longmapsto \mathfrak{S}_{n}\left(\gamma_{1}, \cdots, \gamma_{n}\right)
$$

which satisfy the analogous axioms as the usual Schwinger distributions do, namely the reflexion positivity and the symmetry. However, 
within the analysis of J. Fröhlich, K. Osterwalder and E. Seiler [8, 23], the correlation function may have singularities in those points where two loops intersect and there are some additional technical conditions assumed which are related to the behavior of these singularities. He has proven (compare also [6]) that one can reconstruct from the euclidean correlation functions $\mathfrak{S}_{n}$ an operator valued function $\gamma \mapsto \mathbf{w}_{\gamma}$ together with a unitary strongly continuous representation of $\mathrm{P}_{+}^{\uparrow}$ on $\mathcal{H}$ [8]. Here $\mathbf{w}_{\gamma}$ is only defined for loops which are contained in some space-like plane and it fulfills the covariance condition (1). E. Seiler [23] has also discussed an idea how to proof locality (2). We shall come back to this point later.

For our purpose, we look from an algebraic point of view at the problem of reconstructing a quantum field theory from euclidean data. Let us consider functions

$$
a: \mathcal{A}_{E} \ni u \longmapsto a^{\circ}\left(\int \mathrm{d} x w_{\gamma_{j}+x}(u) f_{j}(x) ; j=1, \cdots, n\right)
$$

on the space of smooth connections $\mathcal{A}_{E}$ in a vector bundle $E$ over the euclidean space $\mathbb{R}^{d}$ where $a^{\circ}$ is a bounded function on $\mathbb{R}^{n}$. These functions are bounded and thus they generate an abelian $\mathrm{C}^{*}$-algebra $A$ with $\mathrm{C}^{*}$-norm

$$
\|a\|=\sup _{u \in \mathcal{A}_{E}}|a(u)|
$$

We assign to a given bounded region $\mathcal{U} \subset \mathbb{R}^{d}$ the $\mathrm{C}^{*}$-sub-algebra $A(\mathcal{U}) \subset A$ which is generated by all functions of Wilson loop variables $w_{\gamma}(f)$ with $\gamma+\operatorname{supp}(f) \subset \mathcal{U}$. The euclidean group $\mathrm{E}(d)$ acts naturally by automorphisms on $A$, namely the prescription

$$
\alpha_{g}: a \longmapsto a \circ g^{-1}: u \longmapsto a(u \circ g)
$$

defines for each $g \in \mathrm{E}(d)$ an appropriate automorphism of $A$, which, of course, acts covariantly on the isotonous net

$$
\underline{A}: \mathcal{U} \longmapsto A(\mathcal{U})
$$

namely we have: $\alpha_{g} A(\mathcal{U})=A(g \mathcal{U})$.

Motivated by the work of E. Seiler, J. Fröhlich and K. Osterwalder [23, 6, 8] as well as that of A. Ashtekar and J. Lewandowski [1], we propose to consider reflexion positive functionals on $A$, i.e. linear 
functionals $\eta \in A^{*}$ which fulfill conditions, corresponding to the axioms (E0)-(E2) above. These functionals can be interpreted as the analogue of the functional integral. Note, if $\eta$ is a state, then $\eta$ is nothing else but a measure on the spectrum $X$ of the $\mathrm{C}^{*}$-algebra $A$. The advantage of this point of view is based on the fact that abelian $\mathrm{C}^{*}$-algebras are rather simple objects namely algebras of continuous functions on a (locally)-compact Hausdorff space.

Overview. In order to make the comprehension of the subsequent sections easier, we shall give an overview of the content of our paper by stating the main ideas and results. This paragraph is also addressed to quick readers who are not so much interested into technical details.

Motivated by the considerations above, we make in Section 2 a suggestion for axioms which an euclidean field theory should satisfy. We start from an isotonous net

$$
\underline{A}: \mathcal{U} \longmapsto A(\mathcal{U}) \subset A
$$

of $\mathrm{C}^{*}$-algebra on which the euclidean group $\mathrm{E}(d)$ acts covariantly by automorphisms of $\alpha: \mathrm{E}(d) \rightarrow$ Aut $A$, like in the example of Wilson loop variables given in the previous paragraph. However, we assume a somewhat weaker condition than commutativity for $A$. For our considerations we only have to assume that two operators commute if they are localized in disjoint regions. In addition to that, we consider a reflexion positive functional $\eta$ on $A$. We shall call the triple $(\underline{A}, \alpha, \eta)$, consisting of the net $\underline{A}$ of $\mathrm{C}^{*}$-algebra, the action of the euclidean group $\alpha$, and the reflexion positive functional, an euclidean field.

We show in Section 3 how to construct from a given euclidean field a quantum field theory in a particular vacuum representation. In order to point out the relation between the euclidean field $(\underline{A}, \alpha, \eta)$ and the Minkowskian world, we briefly describe the construction of a Hilbert space $\mathcal{H}$ on which the reconstructed physical observables are represented. According to our axioms, the map

$$
a \otimes b \longmapsto<\eta, \iota_{e}\left(a^{*}\right) b>
$$

is a positive semidefinite sesqui-linear form on the algebra $A(e)$ of operators which are localized in $e \mathbb{R}_{+}+\Sigma_{e}$ where $\Sigma_{e}$ is the hyper plane orthogonal to the euclidean time direction $e \in S^{d-1}$. Here $\iota_{e}$ is the automorphism on $A$ which corresponds to the reflexion $e \mapsto-e$. By dividing the null-space and taking the closure we obtain a Hilbert 
space $\mathcal{H}$. The construction of the observables, which turn out to be bounded operators on $\mathcal{H}$, is based on two main steps.

Step 1: In Section 3.1, we reconstruct a unitary strongly continuous representation of the Poincaré group $U$ on $\mathcal{H}$. To carry through this analysis, it is not necessary to impose new ideas. The construction is essentially analogous to those which has been presented in [8] (compare also 23]). In order to keep the present paper self contained, we feel obliged to discuss this point within our context in more detail.

Step 2: We discuss in Section 3.2 the construction of the physical observables. At the moment this can only be done, if we assume that the algebra $A$ contains operators which are localized at sharp times, i.e. we require that the algebra $A(e) \cap A(-e)$ is larger than $\mathbb{C} 1$. We shall abbreviate this condition by (TZ) which stands for time-zero. For the fix-point algebra $B(e)$ of $\iota_{e}$ in $A(e) \cap A(-e)$ we obtain a *representation $\pi$ on $\mathcal{H}$, where an operator $\pi(b), b \in B(e)$, is given by the prescription

$$
\pi(b) \mathrm{p}(a) \longmapsto \mathrm{p}(b a)
$$

Here $\mathrm{p}$ is the quotient map, identifying an operator $a \in A(e)$ with its equivalence class $\mathrm{p}(a)$ in $\mathcal{H}$. Now, we consider for a given Poincaré transform $g \in \mathrm{P}_{+}^{\uparrow}$ and a given time-zero operator $b \in B(e)$ the following bounded operator:

$$
\Phi(g, b):=U(g) \pi(b) U(g)^{*} .
$$

We shall say that $\Phi(g, b)$ is localized in a region $\mathcal{O}$ in Minkowski space if $b$ is localized in $\mathcal{U} \subset \Sigma_{e}$ and the transformed region $g \mathcal{U}$ is contained in the double cone $\mathcal{O}$. Let us denote the $\mathrm{C}^{*}$-algebra which is generated by all operators $\Phi(g, b)$, which are localized in $\mathcal{O}$, by $\mathfrak{A}(\mathcal{O})$. Hence we get an isotonous net of $\mathrm{C}^{*}$-algebras

$$
\underline{\mathfrak{A}}: \mathcal{O} \longmapsto \mathfrak{A}(\mathcal{O})
$$

indexed by double cones in Minkowski space on which the Poincaré group acts covariantly by the automorphisms $\alpha_{g}:=\operatorname{Ad}(U(g)), g \in \mathrm{P}_{+}^{\uparrow}$.

The main result: 
(1) The reconstructed isotonous net $\underline{\mathfrak{A}}$ is a Haag-Kastler net: locality holds, i.e. if $\mathcal{O}, \mathcal{O}_{1}$ are two double cones such that $\mathcal{O} \subset \mathcal{O}_{1}^{\prime}$ then $\left[\mathfrak{A}(\mathcal{O}), \mathfrak{A}\left(\mathcal{O}_{1}\right)\right]=\{0\}$.

(2) Furthermore, the $\mathrm{P}_{+}^{\uparrow}$-invariant vector $\Omega=\mathrm{p}(\mathbf{1})$ induces a vacuum state

$$
\omega: a \longmapsto\langle\omega, a\rangle:=\langle\Omega, a \Omega\rangle .
$$

The non trivial aspect of this statement is the proof of locality. As already mentioned above, E. Seiler has discussed an idea how to prove locality for a net of Wilson loops $\mathbf{w}_{\gamma}$. This idea does not rely on the fact that one considers loops. It can also be used for general euclidean fields. However, we have not found a complete proof within the common literature and therefore, which is also one purpose of our paper, we shall present a complete proof here (Section 3.2). The prove is based on the analytic properties of the functions

$$
\begin{aligned}
& F\left(z_{1}, z_{2}\right):=\left\langle\psi, \Phi_{X_{1}}\left(z_{1}, b_{1}\right) \Phi_{X_{2}}\left(z_{2}, b_{2}\right) \hat{\psi}\right\rangle \\
& \hat{F}\left(z_{1}, z_{2}\right):=\left\langle\psi, \Phi_{X_{2}}\left(z_{2}, b_{2}\right) \Phi_{X_{1}}\left(z_{1}, b_{1}\right) \hat{\psi}\right\rangle .
\end{aligned}
$$

We have introduced the operators

$$
\Phi_{X}(z, b):=U(\exp (z X)) \pi(b) U(\exp (-z X))
$$

where $b \in B(e)$ is a time-zero operator and $X$ is a Boost generator or $\mathrm{i} H$ where $H$ is the hamiltonian with respect to the time direction $e$.

Roughly, the argument for the proof of locality goes as follows: Suppose $b_{j}$ is localized in $\mathcal{U}_{j} \subset \Sigma_{e}$. We shall show that the regions $G$ $(\hat{G})$ in which $F(\hat{F})$ are holomorphic are

(a) connected and they contain pure imaginary points $\left(\mathrm{i} s_{1}, \mathrm{i} s_{2}\right)$ and

(b) the intersection $G \cap \bar{G}$ contains all those points $\left(t_{1}, t_{2}\right)$ for which $\mathcal{O}_{1}=\exp \left(t_{1} X\right) \mathcal{U}_{1}$ and $\mathcal{O}_{2}=\exp \left(t_{2} X_{2}\right) \mathcal{U}_{2}$ are space-like separated.

But $F$ and $\hat{F}$ coincide in the pure imaginary points since operators which are localized in disjoint regions commute. This implies

$$
\left.F\right|_{G \cap \hat{G}}=\left.\hat{F}\right|_{G \cap \hat{G}}
$$

and thus by (b) we conclude

$$
\left\langle\psi,\left[\Phi_{X_{1}}\left(t_{1}, b_{1}\right), \Phi_{X_{2}}\left(t_{2}, b_{2}\right)\right] \hat{\psi}\right\rangle=0
$$


if $\Phi_{X_{1}}\left(t_{1}, b_{1}\right)$ and $\Phi_{X_{2}}\left(t_{2}, b_{2}\right)$ are localized in space-like separated regions. We claim that the regions $G$ and $\hat{G}$ depend on the choice of the vector $\hat{\psi}$. However, one can find a dense sub-space $D$ such that $F(\hat{F})$ are holomorphic in $G(\hat{G})$ for all $\hat{\psi} \in D$. Thus the commutator $\left[\Phi_{X_{1}}\left(t_{1}, b_{1}\right), \Phi_{X_{2}}\left(t_{2}, b_{2}\right)\right]$ vanishes on a dense sub-space and, since $\Phi_{X}(t, b)$ is bounded for real points $t \in \mathbb{R}$, the commutator vanishes on $\mathcal{H}$.

In order to get analyticity of $F$ within a region $G$ which is large enough, we prove in the appendix an statement which is the analogue of the famous Bargmann-Hall-Wightman theorem [15, 16, 25].

In Section 4, we discuss some miscellaneous consequences of our result. Note, that for the application of our reconstruction scheme it was crucial to assume that the there are non-trivial euclidean operators which can be localized at sharp times. We shall give some remarks on the condition (TZ) in Section 4.1.

Our considerations can easily be generalized to the case in which there are also fermionic operators present or even though for supersymmetric theories. Here one starts with an isotonous net $\underline{F}: \mathcal{U} \mapsto$ $F(\mathcal{U})$ of $\mathbb{Z}_{2}$-graded $\mathrm{C}^{*}$-algebras which fulfills the time-zero condition (TZ), i.e. the fix-point algebra $B(e)$ of $\iota_{e}$ in $F(e) \cap F(-e)$ is larger than $\mathbb{C} 1$. The euclidean group acts covariantly by automorphisms on $F$ and we require that the graded commutator $[a, b]_{g}=0$ vanishes if $a$ and $b$ are localized in disjoint regions.

Let $\eta$ be a reflexion positive functional, then, by replacing the commutator by the graded commutator, we conclude that the operators

$$
\Phi(g, b)=U(g) \pi(b) U(g)^{*} \quad ; b \in B(e) \text { and } g \in \mathrm{P}_{+}^{\uparrow}
$$

generate a fermionic net $\underline{\mathfrak{F}}$ of $\mathrm{C}^{*}$-algebras. This can really be done analogously to the construction of the Haag-Kastler net $\underline{\mathfrak{A}}$, described above.

Finally, we close our paper by the Section 5 conclusion and outlook.

\section{Axioms for euclidean field theories}

In the present section we make a suggestion for axioms which an euclidean field theory should satisfy.

In the first step, we introduce the notion of an euclidean net of $C^{*}$ algebras. Within our interpretation this notion is related to physical observations. 
Definiton 2.1 : A $d$-dimensional euclidean net of $\mathrm{C}^{*}$-algebras is given by a pair $(\underline{A}, \alpha)$ which consists of an isotonous net

$$
\underline{A}: \mathbb{R}^{d} \supset \mathcal{U} \longmapsto A(\mathcal{U})
$$

of $\mathrm{C}^{*}$-algebras, indexed by bounded subsets in $\mathbb{R}^{d}$ and a group homomorphism $\alpha \in \operatorname{Hom}(\mathrm{E}(d), \operatorname{Aut}(A))$. 1 We require that the pair fulfills the conditions:

(1) Locality: $\mathcal{U}_{1} \cap \mathcal{U}_{2}=\emptyset$ implies $\left[A\left(\mathcal{U}_{1}\right), A\left(\mathcal{U}_{2}\right)\right]=\{0\}$.

(2) Euclidean covariance: $\alpha_{g} A(\mathcal{U})=A(g \mathcal{U})$ for each $\mathcal{U}$.

For an euclidean direction $e \in S^{d-1}$ we consider the reflection $\theta_{e}: e \mapsto-e$. and the sub-group $\mathrm{E}_{e}(d-1)$ which commutes with $\theta_{e}$. Moreover, we set $\iota_{e}:=\alpha_{\theta_{e}}$. As in the introduction, we denote by $A(e)$ the $\mathrm{C}^{*}$-algebra $A\left(e \mathbb{R}_{+}+\Sigma_{e}\right)$ where $\Sigma_{e}$ is the hyper-plane orthogonal to $e$.

Now we formulate a selection criterion for linear functionals on $A$ which corresponds to the selection criterion for physical states. We shall see that class of functional, which is introduced below, is the euclidean analogue of the set of vacuum states.

Definiton 2.2 : We define $S(A, \alpha)$ to be the set of all continuous linear functionals $\eta$ on $A$ which fulfill the following conditions:

(1) e-reflexion positivity: There exists a euclidean direction $e \in S^{d-1}$ such that

$$
\forall a \in A(e): \quad\left\langle\eta, \iota_{e}\left(a^{*}\right) a>\quad \geq 0 .\right.
$$

(2) Unit preserving: $\langle\eta, \mathbf{1}\rangle=1$

(3) Invariance: $\forall g \in \mathrm{E}(d): \eta \circ \alpha_{g}=\eta$.

Remark: We easily observe that the definition of $S(A, \alpha)$ is independent of the chosen direction $e$. In the subsequent, we call the functionals in $S(A, \alpha)$ reflexion positive.

For our purpose it is necessary to require a further condition for the functionals under consideration.

\footnotetext{
${ }^{3}$ We denote the the $\mathrm{C}^{*}$-inductive limit of $\underline{A}$ by $A$. For an unbounded region $\Sigma$ the algebra $A(\Sigma)$ denotes the $\mathrm{C}^{*}$-sub-algebra which is generated by the algebras $A(\mathcal{U}), \mathcal{U} \subset \Sigma$.
} 
Definiton 2.3 : We denote by $S_{R}(A, \alpha)$ the set of all reflexion positive functionals $\eta$ of $A$ for which the map

$$
\mathrm{E}(d) \ni g \longmapsto<\eta, a\left(\alpha_{g} b\right) c>
$$

is a continuous function for each $a, b, c \in A$. These functionals are called regular reflexion positive.

We shall call a triple $(\underline{A}, \alpha, \eta)$ which consists of an euclidean net and a regular reflexion positive functional $\eta$ an euclidean field.

As already mentioned in the introduction, we have to assume that the operators of the euclidean net can be localized at a sharp $d-1$ dimensional hyper plane. For an euclidean time direction $e$ we denote by $B(e)$ the fix-point algebra of $A(e) \cap A(-e)$ under the reflexion $\iota_{e}$.

Condition (TZ): A $d$-dimensional euclidean net of $\mathrm{C}^{*}$-algebras $(\underline{A}, \alpha)$ fulfills the time-zero condition (TZ) iff $B(e)$ is a non-trivial $\mathrm{C}^{*}$ algebra, i.e. it is not $\mathbb{C} \mathbf{1}$. We call the algebras $B(e)$ time-zero algebras. For a region $\mathcal{U} \subset \Sigma_{e}$, we denote by $B(e, \mathcal{U})$ the sub-algebra which is generated by operators localized in $\mathcal{U}$.

Remark: Let $(\underline{A}, \alpha)$ be a $d$-dimensional euclidean net of $\mathrm{C}^{*}$-algebras which fulfill the condition (TZ). Then the net

$$
\underline{B}^{e}: \Sigma_{e} \supset \mathcal{U} \longmapsto B(e, \mathcal{U})
$$

together with the group homomorphism $\beta^{e}:=\left.\alpha\right|_{E_{e}(d-1)}$ is, of course, a $d$-1-dimensional euclidean net of $\mathrm{C}^{*}$-algebras.

\section{From euclidean field theory to quan- tum field theory}

In the present section, we discuss how to pass from a euclidean field $(\underline{A}, \alpha, \eta)$ to a quantum field theory in a particular vacuum representation.

In the first step we construct from a given euclidean field $(\underline{A}, \alpha, \eta)$ a unitary strongly continuous representation of the Poincaré group (Section 3.1).

In the second step we have to require that condition (TZ) is satisfied in order to show that a concrete Haag-Kastler can be reconstructed from the elements of the time-zero algebras and the representation of the Poincaré group (Section 3.2). 


\subsection{Reconstruction of the Poincaré group}

For $e \in S^{d-1}$ we introduce a positive semidefinite sesqui-linear form on $A(e)$ as follows:

$$
a \otimes b \longmapsto<\eta, \iota_{e}\left(a^{*}\right) b>
$$

Its null space is given by

$$
N(e, \eta):=\left\{a \in A(e) \mid \forall b \in A(e):<\eta, \iota_{e}\left(a^{*}\right) b>=0\right\}
$$

and we obtain a pre-Hilbert space

$$
D(e, \eta):=A(e) / N(e, \eta)
$$

The corresponding quotient map is denoted by

$$
\mathrm{p}_{(e, \eta)}: A(e) \longrightarrow D(e, \eta)
$$

and its closure $\mathcal{H}(e, \eta)$ is a Hilbert space with scalar product

$$
\left\langle\mathrm{p}_{(e, \eta)}(a), \mathrm{p}_{(e, \eta)}(b)\right\rangle:=<\eta, \iota_{e}\left(a^{*}\right) b>.
$$

Lemma 3.1 : The map

$$
T_{(e, \eta)}: s \in \mathbb{R}_{+} \longmapsto T_{(e, \eta)}(s): \mathrm{p}_{(e, \eta)}(a) \longmapsto \mathrm{p}_{(e, \eta)}\left(\alpha_{(1, s e)} a\right)
$$

is a strongly continuous semi-group of contractions with a positive generator $H_{(e, \eta)} \geq 0$.

Proof. Since

$$
<\eta, \iota_{e}\left(b^{*}\right) a>=0
$$

for each $b \in A(e)$ implies

$$
<\eta, \iota_{e}\left(b^{*}\right) \alpha_{s e} a>=<\eta, \iota_{e}\left(\alpha_{s e} b^{*}\right) a>=0
$$

for each $b \in A(e)$, we conclude that

$$
T_{(e, \eta)}(s) \mathrm{p}_{(e, \eta)}(a)=0
$$

for $a \in N(e, \eta)$. Hence $T_{(e, \eta)}$ is well defined. The fact that $T_{(e, \eta)}$ is a semi-group of contractions follows by standard arguments, i.e. a 
multiple application of the Cauchy-Schwartz inequality. Finally, the strong continuity follows from the regularity of $\eta$.

We consider the set $\operatorname{Con}(e)$ of all cones $\Gamma$ (in euclidean space) of the form $\Gamma=\mathbb{R}_{+}\left(B_{d}(r+e)\right)+\epsilon e$ where $B_{d}(r)$ denotes the ball in $\mathbb{R}^{d}$ with center $x=0$ and radius $r$. In addition, we define the following subspace of $\mathcal{H}(e, \eta)$

$$
D(\Gamma ; \eta):=\mathrm{p}_{(e, \eta)} A(\Gamma)
$$

Lemma 3.2 : For each cone $\Gamma \in \operatorname{Con}(e)$, the vector space $D(\Gamma, \eta)$ is a dense subspace of $\mathcal{H}(e, \eta)$.

Proof. Lemma 3.1 states that $T_{(e, \eta)}$ is a semi-group of contractions with a positive generator. Furthermore, $D(\Gamma, \eta)$ is mapped into itself by $T_{(e, \eta)}(s)$. Since for each operator $a \in A(e)$ there exists an $s>0$ such that

$$
T_{(e, \eta)}(s) \mathrm{p}_{(e, \eta)}(a) \in D(\Gamma, \eta),
$$

we can apply a Reeh-Schlieder argument in order to prove that $D(\Gamma, \eta)$ is a dense subspace of $\mathcal{H}(e, \eta)$.

Lemma 3.3 : Let $\mathcal{V} \subset \mathrm{E}(d)$ be a small neighborhood of the unit element $1 \in \mathrm{E}(d)$ and let $\Gamma \in \operatorname{Con}(e)$ be a cone such that $\mathcal{V} \Gamma \subset$ $e \mathbb{R}_{+}+\Sigma_{e}$. Then $a \in A(\Gamma) \cap N(e, \eta)$ implies $\alpha_{g} a \in N(e, \eta)$ for each $g \in \mathcal{V}$.

Proof. We have $\left\langle\eta, \iota_{e}\left(b^{*}\right) \alpha_{s e} a\right\rangle=0$ for each $b \in A(\Gamma)$ and hence $<\eta, \iota_{e}\left(b^{*}\right) \alpha_{g} a>=<\eta, \iota_{e}\left(\alpha_{\theta_{e} g} b^{*}\right) a>=0$. Since we may choose $\mathcal{V}$ to be

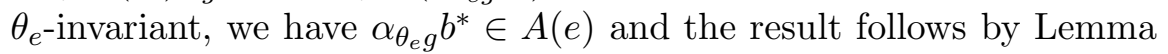
3.2 .

Theorem 3.4 : Let $\eta \in S_{R}(A, \alpha)$ be a regular reflexion positive functional. Then for each $e \in S^{d-1}$ there exists a unitary strongly continuous representation $U_{(e, \eta)}$ of the d-dimensional Poincaré group $\mathrm{P}_{+}^{\uparrow}$

$$
U_{(e, \eta)} \in \operatorname{Hom}\left[\mathrm{P}_{+}^{\uparrow}, U(\mathcal{H}(e, \eta))\right]
$$

such that the spectrum of the translations $x \rightarrow U_{(e, \eta)}(1, x)$ is contained in the closed forward light cone $\bar{V}_{+}$. 
Proof. The theorem can be proven by using the proof of 23, Theorem 8.10]. We briefly illustrate the construction of the representation $U_{(e, \eta)}$. Let $\mathcal{V} \subset \mathrm{E}(d)$ be a small neighborhood of the unit element $1 \in \mathrm{E}(d)$. Then there exists a cone $\Gamma \in \operatorname{Con}(e)$ such that $\mathcal{V} \Gamma \subset e \mathbb{R}_{+}+\Sigma_{e}$. According to Lemma 3.3 we may define for each $g \in \mathcal{V}$ the operator

$$
V_{(e, \eta)}(g) \mathrm{p}_{(e, \eta)}(a):=\mathrm{p}_{(e, \eta)}\left(\alpha_{g} a\right)
$$

with domain $D(\Gamma, \eta)$. If $g$ belongs to the group $\mathrm{E}_{e}(d-1)$ then we conclude that $V_{(e, \eta)}(g)=U_{(e, \eta)}(g)$ is a unitary operator.

Let $\mathfrak{e}(d)$ be the Lie algebra of $\mathrm{E}(d)$ and let $\mathfrak{e}_{e}(d-1) \subset \mathfrak{e}(d)$ be the sub-Lie algebra of $\mathrm{E}_{e}(d-1) \subset \mathrm{E}(d)$. We decompose $\mathfrak{e}(d)$ as follows:

$$
\mathfrak{e}(d)=\mathfrak{e}_{e}(d-1) \oplus \mathfrak{m}_{e}(d-1)
$$

and we obtain another real Lie algebra:

$$
\mathfrak{p}(d):=\mathfrak{e}_{e}(d-1) \oplus \mathfrak{i m}_{e}(d-1)
$$

which is the Lie algebra of the Poincaré group $\mathrm{P}_{+}^{\uparrow}$.

For each $X \in \mathfrak{m}_{e}(d-1)$ there exists a self adjoint operator $L_{(e, \eta)}(X)$ where $D(\Gamma, \eta)$ consists of analytic vectors for $L_{(e, \eta)}(X)$ and for each $s \in \mathbb{R}$ with $\exp (s X) \in \mathcal{V}$ we have:

$$
V_{(e, \eta)}(\exp (s X))=\exp \left(s L_{(e, \eta)}(X)\right) .
$$

According to [23, Theorem 8.10] we conclude that the unitary operators

$$
\begin{array}{r}
U_{(e, \eta)}(\exp (\mathrm{i} s X)):=\exp \left(\mathrm{i} s L_{(e, \eta)}(X)\right) \quad ; \quad X \in \mathfrak{m}_{e}(d-1) \\
U_{(e, \eta)}(g):=V_{(e, \eta)}(g) \quad ; \quad g \in \mathrm{E}_{e}(d-1)
\end{array}
$$

induce a unitary strongly continuous representation of the Poincaré group $\mathrm{P}_{+}^{\uparrow}$. The positivity of the Energy follows from the positivity of the transfer matrix $T_{(e, \eta)}(1)$.

Remark: The vector $\Omega_{(e, \eta)}:=\mathrm{p}_{(e, \eta)}(\mathbf{1})$ is invariant under the action of the Poincaré group. 


\subsection{Reconstruction of the net of local observ- ables}

In the subsequent, we consider a euclidean net of $\mathrm{C}^{*}$-algebras $(\underline{A}, \alpha)$ which fulfills the condition (TZ).

Proposition 3.5 : Let $\eta$ be a regular reflexion positive functional on A. Then the map

$$
\pi_{(e, \eta)}: B(e) \ni b \longmapsto \pi_{(e, \eta)}(b): \mathrm{p}_{(e, \eta)}(a) \longmapsto \mathrm{p}_{(e, \eta)}(b a)
$$

is a well defined ${ }^{*}$-representation of $B(e)$.

Proof. For each $a \in N(e, \eta)$ and for each $c \in A(e)$ we have

$$
<\eta, \iota_{e}\left(c^{*}\right) b a>=<\eta, \iota_{e}\left(c^{*} b\right) a>=0
$$

and hence $\pi_{(e, \eta)}(b)$ is a well defined linear and bounded operator. By construction it is clear that $\pi_{(e, \eta)}$ is a *-homomorphism.

Remark: The restriction of $\left.\eta\right|_{B(e)}$ is a state of $B(e)$. Of course, the GNS-representation of $\left.\eta\right|_{B(e)}$ is a sub-representation of $\pi_{(e, \eta)}$.

\section{Definiton 3.6 :}

(1) Let $\mathcal{O}$ be a double cone in $\mathbb{R}^{d}$. Then we define $\mathfrak{A}_{(e, \eta)}(\mathcal{O})$ to be the $\mathrm{C}^{*}$-algebra on $\mathcal{H}(e, \eta)$ which is generated by operators

$$
\Phi_{(e, \eta)}(g, b):=U_{(e, \eta)}(g) \pi_{(e, \eta)}(b) U_{(e, \eta)}(g)^{*}
$$

with $b \in B(e, \mathcal{U}), g \in \mathrm{P}_{+}^{\uparrow}$ and $g \mathcal{U} \subset \mathcal{O}$.

(2) We denote by $\underline{\mathfrak{A}}_{(e, \eta)}$ the net of $\mathrm{C}^{*}$-algebras which is given by the prescription

$$
\underline{\mathfrak{A}}_{(e, \eta)}: \mathcal{O} \longmapsto \mathfrak{A}_{(e, \eta)}(\mathcal{O})
$$

Theorem 3.7 : The pair $\left(\underline{\mathfrak{A}}_{(e, \eta)}, \operatorname{Ad}\left(U_{(e, \eta)}\right)\right)$ is a $\mathrm{P}_{+}^{\uparrow}$-covariant HaagKastler which is represented on $\mathcal{H}(e, \eta)$. 


\section{Remark:}

(1) Note that

$$
\omega_{(e, \eta)}: \mathfrak{A}_{(e, \eta)} \ni a \longmapsto\left\langle\Omega_{(e, \eta)}, a \Omega_{(e, \eta)}\right\rangle
$$

is a vacuum state since $U_{(e, \eta)}$ is a positive energy representation of the Poincaré group. However, in general $\omega_{(e, \eta)}$ is not a pure state.

(2) For the local algebra $\mathfrak{A}_{(e, \eta)}(\mathcal{O})$, we do not take the von Neumann algebra generated by the corresponding operators $\Phi_{(e, \eta)}(g, b)$ since this might to problems with locality.

Preparation of the proof of Theorem 3.7: For a Lie algebra element $X \in \operatorname{im}_{e}(d-1)$ and a complex number $z \in \mathbb{C}$ we define a linear (unbounded) operator on $\mathcal{H}(e, \eta)$ by

$$
\Phi_{(e, \eta, X)}(z, b):=U_{(e, \eta)}(\exp (z X)) \pi_{(e, \eta)}(b) U_{(e, \eta)}(\exp (-z X))
$$

on a dense domain $D(\Gamma, \eta)$ where $\Gamma \in \operatorname{Con}(e)$ an appropriate cone.

In order to formulate the our next result, we define for two generators $X_{1}, X_{2} \in \operatorname{im}_{e}(d-1)$, for an interval $I$, for a neighborhood $\mathcal{V} \supset \mathrm{L}_{+}^{\uparrow}$ of the unit element in $\mathrm{P}_{+}(\mathbb{C})$, and for two subsets $\mathcal{U}_{j} \subset \Sigma_{e}, j=1,2$, the region

$$
\begin{aligned}
G\left(\mathcal{V} ; X_{1}, X_{2} ; \mathcal{U}_{1}, \mathcal{U}_{2} ; I\right):= & \bigcup_{g \in \mathcal{V} \times \mathrm{L}_{+}^{\uparrow}}\left\{\left(z_{1}, z_{2}\right) \in(\mathbb{R} \times \mathrm{i} I)^{2} \mid \forall \mathbf{x}_{j} \in \mathcal{U}_{j}:\right. \\
& \left.e \operatorname{Im}\left[g\left(\exp \left(z_{1} X_{1}\right) \mathbf{x}_{1}-\exp \left(z_{2} X_{2}\right) \mathbf{x}_{2}\right)\right] \in \mathbb{R}_{+}\right\} .
\end{aligned}
$$

We shall prove in the appendix the lemma given below which is the analogue of the famous BHW theorem (compare also [16, 25] and references given there):

Lemma 3.8 : For a given interval $I$, there exists a dense subspace $D \subset \mathcal{H}(e, \eta)$, such that the function

$F_{\left(X_{1}, X_{2}, b_{1}, b_{2}\right)}:\left(z_{1}, z_{2}\right) \longmapsto\left\langle\psi_{1}, \Phi_{\left(e, \eta, X_{1}\right)}\left(z_{1}, b_{1}\right) \Phi_{\left(e, \eta, X_{2}\right)}\left(z_{2}, b_{2}\right) \psi_{2}\right\rangle$

is holomorphic in $G\left(\mathcal{V} ; X_{1}, X_{2} ; \mathcal{U}_{1}, \mathcal{U}_{2}, I\right)$ for each $\psi_{1}, \psi_{2} \in D$. 
We claim that the $E(d)$ invariance of $\eta$ yields that the dense subspace $D \subset \mathcal{H}(e, \eta)$ can be chosen in such a way that

$$
\begin{aligned}
& \mathrm{I}\left(\mathcal{V} ; X_{2}, X_{2} ; \mathcal{U}_{2}, \mathcal{U}_{1} ; I\right) \\
& :=G\left(\mathcal{V} ; X_{2}, X_{2} ; \mathcal{U}_{2}, \mathcal{U}_{1} ; I\right) \cap G\left(\mathcal{V} ; X_{1}, X_{2} ; \mathcal{U}_{1}, \mathcal{U}_{2} ; I\right) \cap i \mathbb{R}^{2} \\
& \neq \emptyset .
\end{aligned}
$$

Lemma 3.9 : If $\mathcal{U}_{1} \cap \mathcal{U}_{2}=\emptyset$ and $\left(s_{1}, s_{2}\right) \in \mathrm{I}\left(\mathcal{V} ; X_{2}, X_{2} ; \mathcal{U}_{2}, \mathcal{U}_{1} ; I\right)$, then

$$
F_{\left(X_{1}, X_{2}, b_{1}, b_{2}\right)}\left(\mathrm{i} s_{1}, \mathrm{i} s_{2}\right)=F_{\left(X_{2}, X_{1}, b_{2}, b_{1}\right)}\left(\mathrm{i} s_{2}, \mathrm{i} s_{1}\right)
$$

Proof. The lemma is a direct consequence of the euclidean covariance and the locality of the net $\underline{A}$.

Proof of Theorem 3.7: We conclude from Theorem 3.4 and the construction of the algebras $\mathfrak{A}_{(e, \eta)}(\mathcal{O})$ that $\underline{A}_{(e, \eta)}$ is a Poincaré covariant net of $\mathrm{C}^{*}$-algebras, represented on $\mathcal{H}(e, \eta)$.

It remains to be proven that $\mathfrak{A}_{(e, \eta)}$ is a local net. For this purpose it is sufficient to show that for each pair

$$
\begin{aligned}
\left(t_{1}, t_{2}\right) & \in R\left(X_{1}, X_{2} ; \mathcal{U}_{1}, \mathcal{U}_{2}\right) \\
& :=\left\{\left(t_{1}, t_{2}\right) \in \mathbb{R}^{2} \mid \exp \left(t_{1} X_{1}\right) \mathcal{U}_{1} \subset\left(\exp \left(t_{2} X_{2}\right) \mathcal{U}_{2}\right)^{\prime}\right\}
\end{aligned}
$$

the commutator

$$
\left.\left[\Phi_{\left(e, \eta, X_{1}\right)}\left(t_{1}\right), \Phi_{\left(e, \eta, X_{2}\right)}\left(t_{2}\right)\right]\right|_{D}=0
$$

vanishes on an appropriate dense domain $D \subset \mathcal{H}(e, \eta)$.

Since the points in $R\left(X_{1}, X_{2} ; \mathcal{U}_{1}, \mathcal{U}_{2}\right)$ are space-like points, we conclude that there exist complex Lorenz boosts $g_{ \pm} \in \mathcal{V}$ such that

$$
\left.\operatorname{Im} g_{ \pm} R\left(X_{1}, X_{2} ; \mathcal{U}_{1}, \mathcal{U}_{2}\right)\right) \subset V_{ \pm}
$$

Hence we have

$R\left(X_{1}, X_{2} ; \mathcal{U}_{1}, \mathcal{U}_{2}\right) \subset G\left(\mathcal{V} ; X_{1}, X_{2} ; \mathcal{U}_{1}, \mathcal{U}_{2} ; I\right) \cap G\left(\mathcal{V}, X_{2}, X_{1} ; \mathcal{U}_{2}, \mathcal{U}_{1} ; I\right)$ 
Using Lemma 3.9, we conclude that

$$
F_{\left(X_{1}, X_{2}, b_{1}, b_{2}\right)}\left(z_{1}, z_{2}\right)=F_{\left(X_{2}, X_{1}, b_{2}, b_{1}\right)}\left(z_{2}, z_{1}\right)
$$

for

$$
\left(z_{1}, z_{2}\right) \in G\left(\mathcal{V} ; X_{1}, X_{2} ; \mathcal{U}_{1}, \mathcal{U}_{2} ; I\right) \cap G\left(\mathcal{V} ; X_{2}, X_{1} ; \mathcal{U}_{2}, \mathcal{U}_{1} ; I\right)
$$

which finally yields

$$
F_{\left(X_{1}, X_{2}, b_{1}, b_{2}\right)}\left(t_{1}, t_{2}\right)=F_{\left(X_{2}, X_{1}, b_{2}, b_{1}\right)}\left(t_{2}, t_{1}\right)
$$

for each $\left(t_{1}, t_{2}\right) \in R\left(X_{1}, X_{2} ; \mathcal{U}_{1}, \mathcal{U}_{2}\right)$. This proves the locality of $\underline{\mathfrak{A}}_{(e, \eta)}$.

\section{Discussion of miscellaneous conse- quences}

Due to Theorem 3.7 we are able to pass form a euclidean field $(\underline{A}, \alpha, \eta)$ to a quantum field theory in a particular vacuum representation. One crucial condition to apply our method is the existence of the timezero algebras. We shall see that the discussion of Section 4.1 covers all possible situations for euclidean fields which fulfill the condition (TZ).

Afterwards, we discuss in Section 4.2 how the reconstruction scheme has to be generalized in order to include fermionic operators.

\subsection{Some remarks on euclidean fields which satisfy the time-zero condition}

Let us consider a $d$-1-dimensional euclidean net $(\underline{B}, \beta)$ of abelian $\mathrm{C}^{*}$-algebras.

Definiton 4.1 : Let $G$ be a group which contains $\mathrm{E}(d-1)$ as a subgroup. We define $A_{0}(G ; B, \beta)$ to be the ${ }^{*}$-algebra which is generated by pairs $(g, b) \in G \times B$ modulo the relations:

(1) For each $g \in G$, the map $b \longmapsto(g, b)$ is a *-homomorphism.

(2) For each $g \in G$, for each $h \in \mathrm{E}(d-1)$, and for each $b \in B$ : $(g h, b)=\left(g, \beta_{h} b\right)$ 
The algebra $A_{0}(G ; B, \beta)$ possesses a natural $\mathrm{C}^{*}$-norm which is given by

$$
\|a\|:=\sup _{(\mathcal{H}, \pi) \in R(G ; B, \beta)}\|\pi(a)\|_{\mathcal{B}(\mathcal{H})}
$$

where $R(G ; B, \beta)$ is the set of all representations $\pi$ of $A_{0}(G ; B, \beta)$ by bounded operators on a Hilbert space $\mathcal{H}$. The closure of $A_{0}(G ; B, \beta)$ is denoted by $A(G ; B, \beta)$.

Remark: There is a natural group homomorphism

$\alpha \in \operatorname{Hom}(G, \operatorname{Aut} A(G ; B, \beta))$ and a natural faithful embedding $\phi \in$ $\operatorname{Hom}^{*}(B, A(G ; B, \beta))$ given by:

$$
\begin{aligned}
\alpha_{g}\left(g_{1}, b\right) & :=\left(g g_{1}, b\right) \\
\phi(b) & :=(1, b) .
\end{aligned}
$$

Of course, we have for each $h \in \mathrm{E}(d-1)$ :

$$
\phi \circ \beta_{h}=\alpha_{h} \circ \phi
$$

We are mostly interested in two cases for $G$, namely $G=\mathrm{P}_{+}^{\uparrow}$ and $G=\mathrm{E}(d)$. For both groups $A(G ; B, \beta)$ has a natural local structure since $\mathrm{P}_{+}^{\uparrow}$ and $\mathrm{E}(d)$ act as groups on $\mathbb{R}^{d}$.

Definiton 4.2 : For a region $\mathcal{O} \in \mathbb{R}^{d}$ we define $A(G ; B, \beta \mid \mathcal{O})$ to be the $\mathrm{C}^{*}$-sub-algebra in $A(G ; B, \beta)$ which is generated by elements $(g, b)$ with $b \in B(\mathcal{U})$ and $g \mathcal{U} \subset \mathcal{O}$ and we obtain nets

$$
\underline{A}(G ; B, \beta): \mathcal{O} \longmapsto A(G ; B, \beta \mid \mathcal{O}) .
$$

In order to get a Haag-Kastler net for $G=\mathrm{P}_{+}^{\uparrow}$ and a euclidean net for $G=\mathrm{E}(d)$, we consider the following ideals:

(1) $J_{c}\left(\mathrm{P}_{+}^{\uparrow} ; B, \beta\right)$ is the two-sided ideal which is generated by elements $\left[(g, b),\left(g_{1}, b_{1}\right)\right]$ where $(g, b)$ and $\left(g_{1}, b_{1}\right)$ are localized in space like separated regions.

(2) $J_{c}(\mathrm{E}(d) ; B, \beta)$ is the two-sided ideal which is generated by elements $\left[(g, b),\left(g_{1}, b_{1}\right)\right]$ where $(g, b)$ and $\left(g_{1}, b_{1}\right)$ are localized in disjoint regions. 
Thus the prescription

$$
\underline{\mathfrak{A}}_{G}: \mathcal{O} \longmapsto \mathfrak{A}_{G}(\mathcal{O}):=A(G ; B, \beta \mid \mathcal{O}) / J_{c}(G ; B, \beta)
$$

is a $\mathrm{P}_{+}^{\uparrow}$-covariant Haag-Kastler net for $G=\mathrm{P}_{+}^{\uparrow}$, and an euclidean net of $\mathrm{C}^{*}$-algebras for $G=\mathrm{E}(d)$.

Proposition 4.3 : Let $(\underline{A}, \alpha)$ be a d-dimensional euclidean net which fulfills the condition $(T Z)$ and let $(B, \beta)$ be the $d-1$-dimensional euclidean net, corresponding to the hyper plane $\Sigma_{e}$. Then the map

$$
\chi: \mathfrak{A}_{\mathrm{E}(d)} \ni(g, b) \longrightarrow \alpha_{g}(b) \in A
$$

is a *-homomorphism which preserves indeed the net structure.

Proof. By using the relations in Definition 4.1 we conclude, by some straight forward computations, that $\chi$ is a a ${ }^{*}$-homomorphism which preserves the net structure.

An application of Theorem 3.7 gives:

Corollary 4.4 : For each regular reflexion positive functional $\eta$ on $\mathfrak{A}_{\mathrm{E}(d)}$ there exists a vacuum state $\omega_{\eta}$ on $\mathfrak{A}_{\mathrm{P}_{+}^{\uparrow}}$ such that

$$
\left.\omega_{\eta}\right|_{B}=\left.\eta\right|_{B}
$$

\section{Remark:}

(1) Note that we may view $B$ as a common sub algebra of $\mathfrak{A}_{\mathrm{E}(d)}$ and $\mathfrak{A}_{\mathrm{P}_{+}^{\uparrow}}$ since $B \cap J_{c}(G ; B, \beta)=\{0\}$.

(2) Given an euclidean field $(\underline{A}, \alpha, \eta)$, for which the time zero algebra $B:=B(e)$ is non trivial. By Proposition 4.3, we conclude that there is a positive energy representation $\pi_{(e, \eta)}$ of $\underline{\mathfrak{A}}_{\mathrm{P}_{+}^{\uparrow}}$ on the Hilbert space $\mathcal{H}(e, \eta)$ whose image is precisely the net $\underline{\mathfrak{A}}_{(e, \eta)}$. In particular the GNS-representation of $\omega_{\eta}$ is a sub-representation of $\pi_{(e, \eta)}$.

(3) Both, the algebra $\mathfrak{A}_{\mathrm{P}_{+}^{\uparrow}}$ of observables in Minkowski space and the euclidean algebra $\mathfrak{A}_{\mathrm{E}(d)}$ can be considered as sub-algebras of $\mathfrak{A}_{P_{+}(\mathbb{C})}$ where the algebra $\mathfrak{A}_{P_{+}(\mathbb{C})}$ is defined by

$$
\mathfrak{A}_{P_{+}(\mathbb{C})}:=A\left(P_{+}(\mathbb{C}) ; B, \beta\right) /\left[J_{c}\left(\mathrm{P}_{+}^{\uparrow} ; B, \beta\right) \cup J_{c}(\mathrm{E}(d) ; B, \beta)\right] .
$$




\subsection{The treatment of fermionic operators}

In order to discuss the treatment of fermionic operators we introduce the notion of a fermionic euclidean net. The axioms for such a net coincide with those of an euclidean net, except the locality requirement.

Definiton 4.5 : An isotonous and $\mathrm{E}(d)$-covariant net $(\underline{F}, \alpha)$

$$
\underline{F}: \mathbb{R}^{d} \supset \mathcal{U} \longmapsto F(\mathcal{U})=F_{+}(\mathcal{U}) \oplus F_{-}(\mathcal{U})
$$

of $\mathbb{Z}_{2}$-graded $\mathrm{C}^{*}$-algebras is called a fermionic euclidean net iff $\mathcal{U}_{1} \cap$ $\mathcal{U}_{2}=\emptyset$ implies $\left[F\left(\mathcal{U}_{1}\right), F\left(\mathcal{U}_{2}\right)\right]_{g}=\{0\}$, where $[\cdot, \cdot]_{g}$ denotes the graded commutator.

For a given $d$-1-dimensional fermionic net $(\underline{F}, \beta)$, we build the $\mathrm{C}^{*}$ algebras $A(\mathrm{E}(d) ; F, \beta)$ and $A\left(\mathrm{P}_{+}^{\uparrow} ; F, \beta\right)$ as introduced in the previous section. Note, that the algebra $A\left(\mathrm{P}_{+}^{\uparrow} ; F, \beta\right)$ possesses a $\mathbb{Z}_{2}$-grading, namely we have

$$
A\left(\mathrm{P}_{+}^{\uparrow} ; F, \beta\right)=A_{+}\left(\mathrm{P}_{+}^{\uparrow} ; F, \beta\right) \oplus A_{-}\left(\mathrm{P}_{+}^{\uparrow} ; F, \beta\right)
$$

where the algebra $A_{+}\left(\mathrm{P}_{+}^{\uparrow} ; F, \beta\right)$ is spanned by products of elements $(g, b)$ containing an even number of generators in $G \times F_{-}$:

$$
\left(g_{1}, b_{1}\right) \cdots\left(g_{2 n}, b_{2 n}\right)
$$

Therefore the sub-space $A_{-}\left(\mathrm{P}_{+}^{\uparrow} ; F, \beta\right)$ is spanned by elements which are products of elements $(g, b)$ containing an odd number of generators in $G \times F_{-}$:

$$
\left(g_{1}, b_{1}\right) \cdots\left(g_{2 n-1}, b_{2 n-1}\right) .
$$

Analogously to the purely bosonic case, we consider the two-sided ideals

(1) $J_{g}\left(\mathrm{P}_{+}^{\uparrow} ; F, \beta\right)$ which is generated by graded commutators $\left[(g, b),\left(g_{1}, b_{1}\right)\right]_{g}$ where $(g, b)$ and $\left(g_{1}, b_{1}\right)$ are localized in space like separated regions and

(2) $J_{g}(\mathrm{E}(d) ; B, \beta)$ which is generated by graded commutators $\left[(g, b),\left(g_{1}, b_{1}\right)\right]_{g}$ where $(g, b)$ and $\left(g_{1}, b_{1}\right)$ are localized in disjoint regions. 
Thus the prescription

$$
\underline{\mathfrak{F}}_{G}: \mathcal{O} \longmapsto \mathfrak{F}_{G}(\mathcal{O}):=A(G ; F, \beta \mid \mathcal{O}) / J_{g}(G ; F, \beta)
$$

is a fermionic $\mathrm{P}_{+}^{\uparrow}$-covariant Haag-Kastler net for $G=\mathrm{P}_{+}^{\uparrow}$, and an fermionic euclidean net for $G=\mathrm{E}(d)$.

By following the arguments in the proof of Theorem 3.7 and by keeping in mind that the ordinary commutator has to be substituted by the graded commutator, we get the result:

Corollary 4.6 : For each regular reflexion positive functional $\eta$ on the fermionic euclidean net $\mathfrak{F}_{\mathrm{E}(d)}$ there exists a vacuum state $\omega_{\eta}$ on $\mathfrak{F}_{\mathrm{P}_{+}^{\uparrow}}$ such that

$$
\left.\omega_{\eta}\right|_{F}=\left.\eta\right|_{F}
$$

Remark: As described in Section 3.2 the state is defined by

$$
<\omega_{\eta}, \prod_{j=1}^{n}\left(g_{j}, b_{j}\right)>=\left\langle\Omega_{(e, \eta)}, \prod_{j=1}^{n} \Phi_{(e, \eta)}\left(g_{j}, b_{j}\right) \Omega_{(e, \eta)}\right\rangle .
$$

\section{Conclusion and outlook}

\subsection{Concluding remarks and comparison}

We have shown, how a quantum field theory can be reconstructed form a given euclidean field $(\underline{A}, \alpha, \eta)$ which fulfills the condition (TZ). We think, that in comparison to the usual Osterwalder-Schrader reconstruction theorem the reconstruction of a quantum field theory from euclidean fields (in our sense) has the following advantages:

$\oplus$ The Osterwalder-Schrader reconstruction theorem relates Schwinger distributions to a Wightman theory. One obtains an operator valued distribution $\Phi$ which satisfies the Wightman axioms. The reconstructed field operators $\Phi(f)$ are, in general, unbounded operators and in order to get a Haag-Kastler net of bounded operators one has to prove that not only the field operators $\Phi(f), \Phi\left(f_{1}\right)$ commute if $f$ and $f_{1}$ have space-like separated supports, but also its corresponding spectral projections. Furthermore, as mentioned in the introduction, in order to apply the results of [19] one has to prove that the Schwinger distributions are continuous with respect to an appropriate topology. 
Since our considerations are based on $\mathrm{C}^{*}$-algebras, we directly obtain, via our reconstruction scheme, a Haag-Kastler net of bounded operators. In our case, the technical conditions which a reflexion positive functional has to satisfy are more natural. It has to be continuous and regular where the continuity is automatically fulfilled if one considers reflexion positive states.

Our reconstruction scheme does also include objects, like Wilson loop variables, which are not point-like localized objects in a distributional sense. This point of view may also be helpful for constructing gauge theories.

Furthermore, one also may start with an abelian $\mathrm{C}^{*}$-algebra like the example of Wilson loop variables, given in the introduction. Abelian $\mathrm{C}^{*}$-algebras are rather simple objects, namely nothing else but continuous functions on a compact Hausdorff space. In comparison to the construction of reflexion positive functional on the tensor algebra $T_{E}^{\mathcal{T}}(S)$, one may hope that it is easier to construct reflexion positive functionals for abelian $\mathrm{C}^{*}$-algebras. This might simplify the construction of quantum field theory models.

Nevertheless, we also have to mention some drawbacks:

$\ominus$ Unfortunately, our reconstruction scheme is not a complete generalization of the Osterwalder-Schrader reconstruction. This is due to that fact, that we have assumed the existence of enough operators in $A$ which can be localized on a sharp $d$-1-dimensional hyper plane (condition (TZ)). Such a condition is not needed within the OsterwalderSchrader framework and there are indeed examples of quantum field theories which do not fulfill this condition, for instance the generalized free field for which the mass distribution is not $L_{1}$.

On the other hand, the known interacting models like the $P(\phi)_{2}$, the Yukawa 2 as well as the $\phi_{3}^{4}$ model fulfill the condition (TZ). Thus

we think that the existence of the time-zero algebras is not such a harmful requirement.

\subsection{Work in progress}

The main aim of our work in progress is concerned with the construction of examples for euclidean fields which go beyond the free fields.

It would also be desirable to develop a generalization of our reconstruction scheme which also lead directly to a Haag-Kastler net but 
which do not rely on the condition (TZ).

A further open question is concerned with a reconstruction scheme for euclidean fields with cutoffs. The main motivation for such a considerations is based on the work of J. Magnen, V. Rivasseau, and R. Sénéor 17] where it is claimed that the Yang-Mills 4 exists within a finite euclidean volume.

\section{Acknowledgment:}

I am grateful to Prof. Jakob Yngvason for supporting this investigation with many ideas. I am also grateful to Prof. Erhard Seiler and Prof. Jacques Bros for many hints and discussions during the workshop at the Erwin Schrödinger International Institute for Mathematical Physics in Vienna (ESI) this autum. This investigation is financially supported by the Deutsche Forschungsgemeinschaft (DFG) who is also gratefully acknowledged. 


\section{A Analytic properties}

Within this appendix we give a complete proof of Lemma 3.8. We shall use a simplified version of the notation introduced in the previous sections by dropping the indices $(e, \eta)$.

Let $(A, \alpha, \eta)$ be an euclidean field and let $U$ be the corresponding strongly continuous representation of the Poincaré group on $\mathcal{H}=$ $\mathcal{H}(e, \eta)$ which has been constructed by Theorem 3.4. Furthermore, let $\pi$ be the ${ }^{*}$-representation of the time-zero algebra $B$ on $\mathcal{H}$.

For a given tuple $(X, b) \in \operatorname{im}(d-1)^{n} \times B^{n}$, we like to study the analytic properties of the function

$$
\Psi_{n}[X, b]: \mathbb{C}^{2 n} \ni\left(z, z^{\prime}\right) \longmapsto \prod_{j=1}^{n} U_{X_{j}}\left(z_{j}\right) \pi\left(b_{j}\right) U_{X_{j}}\left(z_{j}^{\prime}\right) \psi
$$

where $\psi \in D(\Gamma, \eta)$ and $\Gamma$ is a cone which is contained in $\operatorname{Con}(e)$ and we write:

$$
U_{X}(\zeta):=U(\exp (-\mathrm{i} \zeta X))
$$

For this purpose, we introduce some technical definitions.

Definiton A.1 : For a generator $X \in \operatorname{im}(d-1)$, for an operator $b \in B(\mathcal{U})$ and for a cone $\Gamma \in \operatorname{Con}(e)$, we define the regions:

$$
\begin{aligned}
I(\Gamma, X) & :=\left\{s^{\prime} \mid \exp \left(-\mathrm{i} s^{\prime} X\right) \Gamma \subset e \mathbb{R}_{+}+\Sigma_{e}\right\} \\
J\left(\Gamma, X, b, s^{\prime}\right) & :=\left\{s \mid \exp (-\mathrm{i} s X)\left[\exp \left(-\mathrm{i} s^{\prime} X\right) \Gamma \cup \mathcal{U}\right] \subset e \mathbb{R}_{+}+\Sigma_{e}\right\} \\
G(\Gamma \mid X, b) & :=\bigcup_{s^{\prime} \in I(\Gamma, X)}\left[\mathbb{R}+\mathrm{i} J\left(\Gamma, X, b, s^{\prime}\right) \times \mathbb{R}+\mathrm{i}\left\{s^{\prime}\right\}\right]
\end{aligned}
$$

\section{Definiton A.2 :}

(1) Consider a region $\mathcal{U}$ which is contained in $\Sigma_{e}+e \tau, \tau \geq 0$. We define the corresponding time-zero algebra by $B(\mathcal{U}):=\alpha_{e \tau} B(\mathcal{U}-$ $e \tau)$.

(2) For a given tuple

$$
\left(X, b, s, s^{\prime}\right) \in \operatorname{im}(d-1)^{n} \times B\left(\mathcal{U}_{1}\right) \times \cdots \times B\left(\mathcal{U}_{n}\right) \times \mathbb{R}^{2 n}
$$


we define recursively the regions

$$
\begin{aligned}
\Gamma_{0}:= & \Gamma \\
\Gamma_{1}\left(s_{1}, s_{1}^{\prime}\right):= & \operatorname{conv}\left(\exp \left(-\mathrm{i} s_{1} X_{1}\right)\left[\exp \left(-\mathrm{i} s_{1}^{\prime} X_{1}\right) \Gamma \cup \mathcal{U}_{1}\right]\right) \\
\Gamma_{n}\left(s_{1} \cdots s_{n}, s_{1}^{\prime} \cdots s_{n}^{\prime}\right):= & \operatorname{conv}\left(\operatorname { e x p } ( - \mathrm { i } s _ { n } X _ { n } ) \left[\exp \left(-\mathrm{i} s_{n}^{\prime} X_{n}\right) \times\right.\right. \\
& \left.\left.\times \Gamma_{n-1}\left(s_{1} \cdots s_{n-1}, s_{1}^{\prime} \cdots s_{n-1}^{\prime}\right) \cup \mathcal{U}_{n}\right]\right)
\end{aligned}
$$

Definiton A.3 : For each $n \in \mathbb{N}$ we introduce the region:

$G_{n}(\Gamma ; X, b):=\left\{\left(s_{1} \cdots s_{n}, s_{1}^{\prime} \cdots s_{n}^{\prime}\right) \mid \forall k \leq n: \Gamma_{k}\left(s_{1} \cdots s_{k}, s_{1}^{\prime} \cdots s_{k}^{\prime}\right) \subset e \mathbb{R}_{+}+\Sigma_{e}\right\}$.

Lemma A.4 : For a given tuple

$$
(X, b) \in \operatorname{im}(d-1)^{n} \times B\left(\mathcal{U}_{1}\right) \times \cdots \times B\left(\mathcal{U}_{n}\right)
$$

the function $\Psi_{n}[X, b]$ is holomorphic in $\mathbb{R}^{2 n}+\mathrm{i} G_{n}(\Gamma ; X, b)$.

Proof. We prove the statement by induction. The vector $\psi \in D(\Gamma, \eta)$ is contained in the domain of $U_{X_{1}}\left(\mathrm{i} s_{1}^{\prime}\right)$ as long as $s_{1}^{\prime} \in I\left(\Gamma, X_{1}\right)$. For a fixed value $s_{1}^{\prime} \in I\left(\Gamma, X_{1}\right)$ the vector $\pi\left(b_{1}\right) U_{X_{1}}\left(\mathrm{i} s_{1}^{\prime}\right) \psi$ is contained in the domain of $U_{X_{1}}\left(\mathrm{i} s_{1}\right)$ for $s_{1} \in J\left(\Gamma, X_{1}, b_{1}, s_{1}^{\prime}\right)$. This implies that $\Psi_{1}\left[X_{1}, b_{1}\right]$ is holomorphic in $G\left(\Gamma \mid X_{1}, b_{1}\right) \supset \mathbb{R}+\mathrm{i} G_{1}(\Gamma ; X, b)$.

Suppose $\Psi_{n-1}\left[X_{1} \cdots X_{n-1}, b_{1} \cdots b_{n-1}\right]$ is holomorphic in $\mathbb{R}^{2(n-1)}+$ $\mathrm{i} G_{n-1}(\Gamma ; X, b)$. By the same argument as above we conclude that for a fixed values $\left(s, s^{\prime}\right) \in G_{n-1}(\Gamma ; X, b)$ the function

$$
\left(z_{n}, z_{n}^{\prime}\right) \longmapsto \Psi_{n}[X, b]\left(\mathrm{i} s, z_{n}, \mathrm{i} s^{\prime}, z_{n}^{\prime}\right)
$$

is holomorphic in

$$
G\left(\Gamma_{n-1}\left(s, s^{\prime}\right) \mid X_{n}, b_{n}\right)
$$

and hence it is holomorphic in

$$
\bigcup_{\left(s, s^{\prime}\right) \in G_{n-1}(\Gamma ; X, b)} \mathbb{R}^{2(n-1)}+\mathrm{i}\left\{\left(s, s^{\prime}\right)\right\} \times G\left(\Gamma_{n-1}\left(s, s^{\prime}\right) \mid X_{n}, b_{n}\right)
$$

which is a region containing $G_{n}(\Gamma ; X, b)$. 


\section{B Proof of Lemma 3.8}

For a given euclidean field $(\underline{A}, \alpha, \eta)$ we introduce the following notions:

\section{Definiton B.1 :}

(1) We define the subspace

$$
D(\Gamma ; \eta):=\mathrm{p}_{(e, \eta)} A(\Gamma) \text { and } \hat{D}(\Gamma ; \eta):=U_{(e, \eta)}\left(\mathrm{L}_{+}^{\uparrow}\right) D(\Gamma ; \eta)
$$

(2) Let $X \in \operatorname{im}(d-1)$. For two regions $\Gamma_{1} \subset \Gamma$ we define

$$
I\left(\Gamma_{1}, \Gamma ; X\right):=\left\{s \in \mathbb{R}_{+} \mid \exp (-\mathrm{i} s X) \Gamma_{1} \subset \Gamma\right\} .
$$

(3) For a generator $X \in \mathfrak{i m}(d-1)$ we define the region

$$
\mathcal{U}(s, X):=\exp (-\mathrm{i} s X) \mathcal{U}
$$

for each $s \in \mathbb{R}$.

(4) Given two regions $\mathcal{U}_{1}, \mathcal{U}_{2}$ in $\mathbb{R}^{d}$, we define

$$
\begin{aligned}
G_{e}\left(X_{1}, X_{2} ; \mathcal{U}_{1}, \mathcal{U}_{2} ; I\right):= & \left\{\left(z_{1}, z_{2}\right) \in(\mathbb{R} \times \mathrm{i} I)^{2} \mid \forall \mathbf{x}_{j} \in \mathcal{U}_{j}:\right. \\
& \left.e \operatorname{Im}\left(\exp \left(z_{1} X_{1}\right) \mathbf{x}_{1}-\exp \left(z_{2} X_{2}\right) \mathbf{x}_{2}\right) \in \mathbb{R}_{+}\right\} \\
G_{e}^{g}\left(X_{1}, X_{2} ; \mathcal{U}_{1}, \mathcal{U}_{2} ; I\right):= & \left\{\left(z_{1}, z_{2}\right) \in(\mathbb{R} \times \mathrm{i} I)^{2} \mid \forall \mathbf{x}_{j} \in \mathcal{U}_{j}:\right. \\
& \left.e \operatorname{Im}\left[g\left(\exp \left(z_{1} X_{1}\right) \mathbf{x}_{1}-\exp \left(z_{2} X_{2}\right) \mathbf{x}_{2}\right)\right] \in \mathbb{R}_{+}\right\}
\end{aligned}
$$

where $g \in \mathrm{P}_{+}(\mathbb{C})$ is a complex Poincaré transformation.

Lemma B.2 : Let $\Gamma_{1}, \Gamma \in \operatorname{Con}(e)$ be two conic regions such that $g \Gamma_{1} \subset \Gamma$ is a proper inclusion. Then there exists an interval I such that for each $b_{1} \in B\left(\mathcal{U}_{1}\right), b_{2} \in B\left(\mathcal{U}_{2}\right)$ and for each $\psi_{1}, \psi_{2} \in \hat{D}\left(\Gamma_{1} ; \eta\right)$ the function

$$
F_{\left(X_{1}, X_{2}, b_{1}, b_{2}\right)}^{\left(\psi_{1}, \psi_{2}\right)}:\left(z_{1}, z_{2}\right) \longmapsto\left\langle\psi_{1}, \Phi_{X_{1}}\left(z_{1}, b_{1}\right) \Phi_{X_{2}}\left(z_{2}, b_{2}\right) \psi_{2}\right\rangle
$$

is holomorphic in $G_{e}\left(X_{1}, X_{2} ; \mathcal{U}_{1}, \mathcal{U}_{2} ; I\right)$. 
Proof. First we obtain by an application of Lemma A.4, that for each $\psi_{1} \in \mathcal{H}(e, \eta)$ and for each $\psi \in D(\Gamma, \eta)$, the function

$$
(z, \zeta) \longmapsto\left\langle\psi_{1}, \Phi_{X_{2}}\left(z, b_{2}\right) U_{X}(\zeta) \psi_{2}\right\rangle
$$

is holomorphic for $\operatorname{Im} \zeta \in I\left(\Gamma_{1}, \Gamma ; X\right)$ and $\operatorname{Im} z \in I\left(\Gamma ; X_{2}\right)$. for $X \in$ $\operatorname{im}(d-1)$. The holomorphy is due to the fact that $U$ is a strongly continuous representation of the Poincaré group and that $D(\Gamma ; \eta)$ consists of analytic vectors for the boost generators.

For a fixed values $s^{\prime} \in I\left(\Gamma_{1}, \Gamma ; X\right)$ and $s \in I\left(\Gamma ; X_{2}\right)$, we have

$$
\Phi_{X_{2}}\left(\mathrm{i} s, b_{2}\right) U_{(e, \eta, X)}\left(-\mathrm{i} s^{\prime}\right) \psi_{2} \in D(\hat{\Gamma} ; \eta)
$$

for each region $\hat{\Gamma} \subset e \mathbb{R}_{+}+\Sigma_{e}$ which contains $\Gamma \cup \mathcal{U}_{2}\left(s, X_{2}\right)$.

Now, for a given point $(z, \mathrm{i} s) \in G_{e}\left(X_{1}, X_{2} ; \mathcal{U}_{1}, \mathcal{U}_{2} ; I\right)$ there exists a conic region $\Gamma(z, \mathrm{i} s) \in \operatorname{Con}(e)$ with $\Gamma(z$, is $) \supset \Gamma \cup \mathcal{U}_{2}\left(s, X_{2}\right)$ such that $D(\Gamma(z, \mathrm{i} s) ; \eta)$ is contained in the domain of $\Phi_{X_{1}}\left(z, b_{2}\right)$. Furthermore, for a given interval $I$, the cone $\Gamma$ can be chosen to be small enough such that this holds for each $(z$, is $)$ with $\operatorname{Im} z, s \in I$. Since $\Gamma_{1}$ is $O(d-1)$-invariant, the result follows.

Let $\mathcal{V} \supset \mathrm{L}_{+}^{\uparrow}$ be a neighborhood of the identity in $\mathrm{P}_{+}(\mathbb{C})$. We may choose a cone $C(\Gamma, \mathcal{V}) \in \operatorname{Con}(e)$ such that

$$
g C(\Gamma, \mathcal{V}) \subset \Gamma .
$$

for each $g \in \mathrm{E}(d) \cap \mathcal{V}$. Note that the representation $U$ can be extended to $\mathcal{V}$ by unbounded operators with domain $\hat{D}\left(\Gamma_{1}, \eta\right)$ where $\Gamma_{1} \subset C(\Gamma, \mathcal{V})$.

In order to finish the proof of Lemma 3.8, we show the following statement:

Lemma B.3 : Let $\mathcal{U}_{1}, \mathcal{U}_{2}$ be two bounded disjoint regions and let $\Gamma_{1} \in \operatorname{Con}(e)$ such that $\Gamma_{1} \subset C(\Gamma, \mathcal{V})$ is a proper inclusion. Then the function $F_{\left(X_{1}, X_{2}, b_{1}, b_{2}\right)}^{\left(\psi_{1}, \psi_{2}\right)}$ has an extension $\hat{F}_{\left(X_{1}, X_{2}, b_{1}, b_{2}\right)}^{\left(\psi_{1}, \psi_{2}\right)}$ which is holomorphic in

$$
G\left(\mathcal{V} ; X_{1}, X_{2} ; \mathcal{U}_{1}, \mathcal{U}_{2} ; I\right):=\bigcup_{g \in \mathcal{V}} G_{e}^{g}\left(X_{1}, X_{2} ; \mathcal{U}_{1}, \mathcal{U}_{2} ; I\right)
$$

for each $\psi_{1}, \psi_{2} \in \hat{D}\left(\Gamma_{1} ; \eta\right)$. 
Proof. For a given neighborhood $\mathcal{V} \supset \mathrm{L}_{+}^{\uparrow}$ of the unit element in $\mathrm{P}_{+}(\mathbb{C})$ and for a given cone $\Gamma \in \operatorname{Con}(e)$, there exists $\epsilon>0$ such that $g \mathcal{U}_{2}+\epsilon e \subset \Gamma$. We easily observe that the substitution

$$
\begin{aligned}
\psi_{j}^{\prime} & :=T(\epsilon) U(g) \psi_{j} \\
X_{j}^{\prime} & :=\exp (-\mathrm{i} \epsilon H) g X_{j} g^{-1} \exp (\mathrm{i} \epsilon H)
\end{aligned}
$$

yields

$$
F_{\left(X_{1}^{\prime}, X_{2}^{\prime}, b_{1}, b_{2}\right)}^{\left(\psi_{1}^{\prime}, \psi_{1}^{\prime}\right)}\left(z_{1}, z_{2}\right)=F_{\left(X_{1}, X_{2}, b_{1}, b_{2}\right)}^{\left(\psi_{1}, \psi_{2}\right)}\left(z_{1}, z_{2}\right)
$$

for each $\left(z_{1}, z_{2}\right) \in G_{e}\left(X_{1}, X_{2} ; \mathcal{U}_{1}, \mathcal{U}_{2} ; I\right)$ where $H$ is the generator of translations in $e$-direction. According to Lemma B.2, the function $F_{\left(X_{1}^{\prime}, X_{2}^{\prime}, b_{1}, b_{2}\right)}^{\left(\psi_{1}^{\prime}, \psi^{\prime}\right)}$ is holomorphic in $G_{e}^{g}\left(X_{1}, X_{2} ; \mathcal{U}_{1}, \mathcal{U}_{2} ; I\right)$ which implies the result. 


\section{References}

[1] Ashtekar, A. and Lewandowski, J.:

Differential geometry on the space of connections via graphs and projective limits.

J. Geom. Phys. 17, 191-230, (1995)

[2] Brydges, D.:

A short course on cluster expansions.

In Les Houches 1984, Proceedings, Critical Phenomena, Random Systems, Gauge Theories, 129-183.

[3] Driessler,W. and Fröhlich, J.:

The reconstruction of local algebras from the Euclidean Green's functions of relativistic quantum field theory. Ann. Inst. Henri Poincaré 27, 221-236, (1977)

[4] Driessler, W. and Summers, S.J.:

Nonexistence of quantum fields associated with twodimensional spacelike planes.

Commun. Math. Phys. 89, 221-226, (1983)

[5] Feldman, J. and Osterwalder, K.:

The Wightman axioms and the mass gap for weakly coupled $\phi_{3}^{4}$ quantum field theories.

In: Mathematical Problems in Theoretical Physics, H. Araki, ed., Berlin, Heidelberg, New York: SpringerVerlag.

[6] Fröhlich, J.:

Some results and comments on quantized gauge fields.

Cargese, Proceedings, Recent Developments In Gauge Theories, 53-82, (1979)

[7] Fröhlich, J.:

On the triviality of $\lambda \phi^{4}$ in d-dimensions theories and the approach to the critical point in $d>4$-dimensions.

Nucl. Phys. B200, 281-296, (1982)

[8] Fröhlich, J., Osterwalder, K. and Seiler E.:

On virtual representations of symmetric spaces and their analytic continuation.

Ann. Math. 118, 461-489, (1983) 
[9] Gawedzki, G. and Kupiainen, A.:

Asymptotic freedom beyond perturbation theory.

Les Houches lectures (1984)

[10] Glimm, J. and Jaffe, A.:

Collected Papers. Vol. 1 and Vol.2: Quantum field

therory and statistical mechanics.

Expositions. Boston, USA: Birkhäuser (1985)

[11] Glimm, J. and Jaffe, A.:

$A \lambda \phi^{4}$ quantum field theory without cutoffs I.

Phys. Rev. 176, 1945-1951, (1968)

Glimm, J. and Jaffe, A.:

$A \lambda \phi^{4}$ quantum field theory without cutoffs II.

Ann. of Math. 91, 362-401, (1970)

Glimm, J. and Jaffe, A.:

$A \lambda \phi^{4}$ quantum field theory without cutoffs III.

Acta Math. 125, 204-267, (1970)

Glimm, J. and Jaffe, A.:

$A \lambda \phi^{4}$ quantum field theory without cutoffs $I V$.

J. Math. Phys. 13, 1568-1584, (1972)

[12] Glimm, J. and Jaffe, A.:

The Yukawa-2 quantum field theory without cutoffs.

J. Funct. Anal. 7, 323-357, (1971)

[13] Glimm, J. and Jaffe, A.:

Positivity of the $\phi_{3}^{4}$ hamiltonian.

Fortschritte der Physik 21, 327-376, (1973)

[14] Glimm, J. and Jaffe, A.:

Quantum physics, a functional integral point of view.

Springer, New York, Berlin, Heidelberg (1987)

[15] Hall, D. and Wightman, A.S.:

A theorem on invariant analytic functions with applications to relativistic quantum field theory.

Mat. Fys. Medd. Dan. Vid. Selsk. 31, no.5, (1957)

[16] Jost, R.:

The general theory of quantized fields.

American Mathematical Society, Providence, Rhode Island 1965. 
[17] Magnen, J., Rivasseau, V. and Sénéor, R.:

Construction of YM-4 with an infrared cutoff.

Commun. Math. Phys. 155, 325-384, (1993)

[18] Magnen, J. and Sénéor, R.:

The infinite volume limit of the $\phi_{3}^{4}$ model.

Inst. H. Poincaré 24, 95-159, (1976)

[19] Osterwalder, K. and Schrader, R.:

Axioms for Euclidean Green's functions I.

Commun. Math. Phys. 31, 83-112, (1973)

Osterwalder, K. and Schrader, R.:

Axioms for Euclidean Green's functions II.

Commun. Math. Phys. 42, 281-305, (1975)

[20] Polyakov, A. M.:

Gauge fields as rings of glue.

Nucl. Phys. B164, 171-188, (1979)

[21] Schrader, R.:

A remark on Yukawa plus boson self-interaction in two space-time dimensions.

Commun. Math. Phys. 21, 164-170, (1971)

[22] Schrader, R.:

A Yukawa quantum field theory in two space-time dimensions without cutoffs.

Ann. Phys. 70, 412-457, (1972)

[23] Seiler, E.:

Gauge theories as a problem of constructive quantum field theory and statistical mechanics.

Berlin, Germany: Springer (1982) 192 P. ( Lecture Notes In Physics, 159).

[24] Seiler, E. and Simon, B.:

Nelson's symmetry and all that in the $Y_{u k a w a_{2}}$ and $\phi_{3}^{4}$ field theories.

Ann. Phys. 97, 470-518, (1976)

[25] Streater, R.F. and Wightman, A.S.: PCT, spin and statistics and all that. Redwood City, USA: AddisonWesley (1989) 207 p. (Advanced book classics). 